\title{
O trabalho de enfermagem no Hospital Dia na perspectiva da reforma psiquiátrica em João Pessoa - Paraíba*
}

\author{
NURSING WORK OF NURSING AT A DAY HOSPITAL IN JOÃO PESSOA IN THE \\ PERSPECTIVE OF THE PSYCHIATRIC REFORM
}

\author{
EL TRABAJO DE ENFERMERÍA EN UN HOSPITAL DE DIA EN LA PERSPECTIVA \\ DE LAREFORMA PSIQUIÁTRICA EN JOÃO PESSOA - PARAÍBA
}

\section{Ana Tereza Medeiros C Silva', Sônia Barros²}

\begin{abstract}
RESUMO
Relatamos uma investigação para apreender temas que explicam o trabalho de enfermagem, no Hospital-Dia, na perspectiva da reforma psiquiátrica em João Pessoa-PB. Utilizamos o referencial do materialismo histórico e dialético e o trabalho como categoria analítica. O material empírico foi analisado pela técnica de análise do discurso. $\mathrm{O}$ estudo revelou um tema coincidente, $m u$ dar o modelo de assistência psiquiátrica tradicional, e, nesse sentido, o trabalho de enfermagem incorpora além da qualidade formal, técnica-especializada, uma qualidade política indicando um novo significado ao processo de trabalho de enfermagem.
\end{abstract}

\section{DESCRITORES}

Enfermagem psiquiátrica.

Hospitais-dia.

Desinstitucionalização.

Saúde mental.

\begin{abstract}
This is the report of an investigation that was carried out in order to apprehend themes that explain nursing work at a day hospital in João Pessoa, State of Paraíba, in the perspective of the psychiatric reform. The authors used the referential of dialectical and historical materialism and work as an analytical category. The empirical material was analyzed through the technique of discourse analysis. The study revealed a coincident theme - to change the model of traditional psychiatric assistance - and, in this sense, the work of nursing incorporates, in addition to its formal, technically specialized quality, a political quality, thus indicating a new meaning to the nursing work process.
\end{abstract}

\section{KEY WORDS}

Psychiatric nursing. Hospitals, day.

Desinstitutionalization.

Mental health.

\section{RESUMEN}

Relatamos una investigación para capturar temas que explican el trabajo de enfermería, en un Hospital de Día, en la perspectiva de la reforma psiquiátrica en João Pessoa-PB. Utilizamos el referencial del materialismo histórico y dialéctico, y el trabajo como categoría analítica. El material empírico fue analizado por la técnica del análisis del discurso. El estudio reveló un tema coincidente, cambiar el modelo de asistencia psiquiátrica tradicional, y en ese sentido el trabajo de enfermería incorpora además de la calidad formal, técnicaespecializada, una calidad política indicando un nuevo significado al proceso de trabajo de enfermería en salud mental.

\section{DESCRIPTORES}

Enfermería psiquiátrica. Hospitales de dia. Desinstitucionalización. Salud mental.

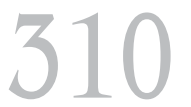




\section{INTRODUÇÃO}

Neste estudo descrevemos o percurso investigativo empreendido em tese de doutoramento, com o objetivo de apreender temas que qualificam o processo de trabalho de enfermagem em saúde mental, no Hospital-Dia, da cidade de João Pessoa-PB, na perspectiva da reforma psiquiátrica proposta no país. Pressupomos que a mudança requerida pela nova política de saúde se materializa nos processos de trabalho institucionais, cuja análise permite revelar as qualidades que a reforma assume para constituir um novo Modelo de Assistência em Saúde Mental. Este corresponde a uma categoria que sintetiza a compreensão do relacionamento dinâmico entre a política, que informa suas diretrizes e o modo de organizar os serviços, onde se pratica o trabalho que deve efetivá-la. Nesse enfoque, o trabalho de enfermagem é entendido como um momento do processo de trabalho para a produção de saúde mental, constituindo parte significativa do trabalho nessa área.

O trabalho humano nas sociedades capitalistas foi estudado por Marx que descreveu seu processo, a partir da relação entre três elementos:

a) a atividade adequada a um fim, isto é o próprio trabalho;

b) a matéria a que se aplica o trabalho, o objeto de trabalho;

c) os meios de trabalho, o instrumental de trabalho ${ }^{(1)}$.

Na perspectiva marxista, compreende-se os processos de trabalho em saúde como um conjunto articulado de momentos indispensáveis ao encadeamento para a produção intencional de respostas a necessidades de saúde ${ }^{(2)}$, a partir da antevisão do potencial para uma transformação mediada por uma energia de atividade que corresponde ao trabalho. Assim, as idéias de necessidade, energia, intencionalidade e transformação de uma realidade, para atender as carências de saúde, sintetizadas em um único processo, representam o conceito do autor sobre o trabalho em saúde. No enfoque, a saúde é compreendida como um conjunto de relações entre o Ser Humano, a Natureza, o Trabalho e a Sociedade - que se revela na constituição e aplicação dos saberes e práticas específicas, constituídas no século XVIII, para manter/recuperar a força produtiva humana desprendida no trabalho, incapacitada pela doença, naquele momento histórico.

Desse modo, o trabalho em saúde pode ser tomado como uma categoria sociológica de análise por sua condição de prática social, na qual o saber sobre o processo de viver, adoecer e morrer constitui-se em um dos elementos do seu processo de trabalho [instrumento], exprimindo não apenas as soluções técnicas pertinentes ao trabalho, mas, indicando também como o ser humano se organiza na produção e reprodução da sua existência.

O trabalho de enfermagem como prática social, e, um dos meios de produção de saúde mental, ao revelar seu saber informa, também, as influências que sofre e as tendências das qualidades que incorpora para atender à nova política de saúde mental, a inclusão social. Essa diretriz da política determina um dos elementos do processo de trabalho em saúde mental, sua finalidade, indicando uma direção oposta à finalidade de segregação do modelo tradicional de assistência psiquiátrica. Na nova perspectiva, todos os elementos do processo de trabalho em saúde mental precisam articular-se para que o trabalho tenha potencial transformador do Modelo Tradicional de Assistência Psiquiátrica, autorizado por saberes construídos em outro contexto para atender necessidades sociais pertinentes àquele momento histórico.

Assim, o trabalho para produzir a finalidade da reforma psiquiátrica, de inclusão social da pessoa com doença mental, assume o estatuto de campo estratégico de mudança, para responder às necessidades sociais que a demandou, pois, a transformação da assistência psiquiátrica anunciada no plano das intenções, precisa ocorrer tanto na maneira de conceber o trabalho, quanto no modo concreto de processá-lo para atingir sua finalidade. Disso decorre a necessidade de se adequarem, coerentemente, os elementos dos processos de trabalho, o objeto e os instrumentos à finalidade pretendida.

$\mathrm{Na}$ atualidade, o trabalho para qualificar uma reforma psiquiátrica transformadora do modelo tradicional precisa rever seus instrumentos, entre os quais o saber da Psiquiatria Tradicional fundada em princípios ontológicos de normalidade-anormalidade, que a caracteriza desde o século XVIII, e marcada pela concepção da saúde/doença mental organizada em torno da exclusividade biológica, enfoque que permitiu a institucionalização da loucura, sobretudo, como doença mental ${ }^{(3)}$.

Todavia, a loucura pode ser compreendida como uma experiência humana, vivida em todas as épocas e interpretada conforme a visão de mundo dominante, nas sociedades de cada momento histórico. Na modernidade, foi incorporada pela medicina no enfoque da doença mental e recortada para ser objeto do trabalho médico, permitindo a organização da Psiquiatria. No século XX, a especiali-
O trabalho de enfermagem no hospital dia na perspectiva da reforma psiquiátrica em João Pessoa Paraíba 
Ana Teresa M.C. Silva Sônia Barros dade foi criticada por seu caráter disciplinador e excludente. A crítica incentivou o surgimento da Psiquiatria Social que recortou um novo objeto de interesse, a saúde mental.

Depois da Segunda Guerra Mundial, o novo enfoque motivou os diversos movimentos por mudanças no modo tradicional de assistência psiquiátrica no mundo, entre os quais as Comunidades Terapêuticas, na Inglaterra; a Psicoterapia Institucional, na França; o movimento da Psiquiatria Preventiva, nos Estados Unidos. Diferentemente desses países, na Itália, o movimento por mudanças na assistência psiquiátrica tradicional apresentou uma crítica às Comunidades Terapêuticas, por essa abordagem permitir a manutenção do asilo e do saber que o autoriza, parecendo uma tentativa de resgatar a positividade do hospital psiquiátrico e de seu caráter de instrumento de cura da doença mental, prometida desde sua fundação e jamais confirmada. Em oposição, o movimento italiano recortou como objeto de seu interesse, não a saúde ou a doença mental, mas, a existência e o sofrimento da pessoa com doença mental ${ }^{(4)}$.

No Brasil, a Psiquiatria sofreu influências de tais movimentos sociais tanto na política de saúde mental, quanto no movimento social que se fortalece, desde a década de 1970, na luta pela democracia no país, cujas propostas foram expressas nas Conferências Nacionais de Saúde Mental ${ }^{(5-7)}$. Assim, o processo de reforma psiquiátrica no país, transcende a busca de soluções apenas técnicas, visando transformar aspectos técnicos, políticos e sociais sobre a loucura e suas instituições. $\mathrm{O}$ avanço nesse sentido tem como horizonte, a desinstitucionalização da doença e do doente mental ${ }^{(8)}$.

Em João Pessoa, desde a década de 1990, organizou-se o Movimento de Luta Antimanicomial que reclama uma reforma psiquiátrica com visibilidade de mudanças transformadoras da assistência psiquiátrica nos seus serviços públicos. Em resposta a essa necessidade, a Secretaria de Saúde do Estado promoveu a reorganização geo-administrativa desses serviços: reuniu todas as instituições psiquiátricas públicas constituindo o Complexo Psiquiátrico Juliano Moreira - CPMJ, no espaço murado da antiga Colônia, sob uma única direção. No mesmo espaço, fundou mais dois novos serviços: o Hospital-Dia HD, inaugurado, em 1998, e o Centro de Atenção Psicossocial - CAPS - Dr. Gutemberg Botelho, inaugurado em abril de 2001.

Os novos tipos de serviços são defendidos pelo Ministério da Saúde como substitutivos do
Hospital Psiquiátrico Tradicional, devendo apresentar possibilidades de promover a inclusão social da pessoa com doença mental. Portanto, são novos instrumentos da atual política para promover a reforma psiquiátrica, que pressupõe um novo modo de organizar e processar o trabalho para produzir saúde mental. A partir dessas reflexões, com este estudo, buscamos identificar na qualidade que o trabalho de Enfermagem do HD de João Pessoa revela, a mudança que o processo de trabalho de enfermagem realiza em relação ao modelo tradicional, para atender à necessidade da reforma psiquiátrica proposta como nova política de saúde mental, na atualidade.

\section{DESCRIÇÃO METODOLÓGICA}

Estudo de abordagem qualitativa que tem referencial teórico-metodológico no Materialismo Histórico e Dialético - MHD, do qual recorta o Trabalho como categoria de análise. A eleição metodológica do MHD justifica-se pela adequação de seus pressupostos ao objeto do estudo, o processo de trabalho de enfermagem e, também, por ser uma abordagem empregada com muita freqüência nos estudos que analisam os fenômenos sociais em transformação, possibilidade que a proposta de uma nova política de saúde mental no país, suscita.

O MHD é constituído por uma teoria crítica da história, o materialismo histórico e por uma teoria filosófica, o materialismo dialético que explicam a realidade social em permanente transformação, movida por contradições, cuja superação é mediada pelo trabalho humano. Na abordagem, a matéria é representada pelas relações sociais, e a dialética é compreendida como o movimento da ação humana que produz a história. Nesse enfoque, o trabalho é uma categoria sociológica de análise reconhecida por seu potencial transformador da realidade para atender necessidades individuais e coletivas ${ }^{(9)}$.

A estratégia da pesquisa consistiu em investigar os elementos constitutivos do processo de trabalho de enfermagem, na posição discursiva dos sujeitos da pesquisa sobre seus trabalhos e sobre o processo da reforma psiquiátrica nacional e local. A técnica de obtenção do material empírico foi a da entrevista. Adotamos como instrumento, um formulário de entrevista semiestruturado - autorizado pelo Comitê de Pesquisa e Ética da EEUSP - com três questões abertas sobre a concepção da reforma psiquiátrica e sobre o trabalho que realizam os sujeitos da pesquisa. 
Depois das entrevistas, realizamos a observação do trabalho de enfermagem, por um período de uma semana em dois turnos, buscando identificar a correspondência, na prática, para as posições defendidas nos discursos, sobre o trabalho de enfermagem. Registramos em um diário de campo as atividades desenvolvidas pela enfermagem; a maneira como elas eram executadas; a clientela atendida; o diálogo estabelecido entre os sujeitos e os instrumentos empregados. Essas anotações ajudaram, também, a compreender a organização do serviço e sua dinâmica.

Entrevistamos duas enfermeiras e duas auxiliares de enfermagem do HD. As entrevistas foram gravadas e identificadas com pseudônimos. Em seguida, elas foram transcritas em sua íntegra, para nelas buscarmos as concepções do processo da reforma psiquiátrica, os elementos do processo trabalho de enfermagem nessa perspectiva, principalmente, o recorte do objeto de trabalho, cuja concepção a enfermagem emprega como saber instrumental.

O material empírico obtido nas entrevistas foi analisado conforme a técnica de análise do discurso $^{(10)}$. Nessa abordagem, o texto é uma organização dotada de mecanismos de coerência nos quais podemos perceber as visões de mundo dos sujeitos, onde se encontram os temas com os quais os pesquisadores trabalham. Assim, enquanto o texto é uma produção individual, o discurso é uma posição social porque materializa na linguagem e veicula na sociedade a representação ideológica do sujeito, inscrita no texto. No enfoque, a identificação de temas ocorre pela análise dos três planos da estrutura do texto: o superficial, de maior concretude; o intermediário, no qual se definem os valores dos sujeitos, e, o plano profundo, o nível mais abstrato, no qual a oposição reguladora dos sentidos pode ser percebida em temas que conferem unidade aos elementos dos diversos planos, e, significado ao todo. A partir da identificação desses temas, os pesquisadores produzem as categorias empíricas de seus estudos.

\section{ANÁLISE E DISCUSSÃO DOS RESULTADOS}

As atividades desempenhadas pelos enfermeiros como trabalho de enfermagem foram relativas à admissão e ao acolhimento dos usuários no serviço; à administração de medicamentos, cuidados com a higiene e alimentação; à participação dos usuários nas atividades programadas no serviço, reuniões, grupos de trabalho; à orientação aos familiares sobre o estado de saúde do usuário e sobre a importância da organização e da participação dos parentes nos movimentos sociais, como também no serviço; ao funcionamento do serviço, atividades administrativas e burocráticas, como distribuição do seu pessoal nos diversos setores, anotações, encaminhamentos de documentos, controle da medicação psicotrópica e relatório de atividades.

Verificamos que a enfermagem desenvolve seu processo de trabalho com dois diferentes objetos inter-relacionados, o processo saúde-doença mental e uma prática educativa para a participação social. Desse modo, a assistência/cuidado de enfermagem em saúde mental revelou-se tanto no âmbito singular da individualidade - respostas a necessidades individuais de saúde mental, quanto no âmbito da Saúde Coletiva - respostas a necessidades sociais relacionadas à saúde mental.

O trabalho de enfermagem que tem por objeto o cuidar individual é desenvolvido, sobretudo, no hospital como parte do trabalho médico que, historicamente, incorporou como seu objeto de trabalho, os corpos individuais ${ }^{(11)}$. Nessa perspectiva a enfermagem desenvolveu seu sistema de assistência, organizado em dois diferentes processos, o Individual e o Coletivo. O processo de trabalho para Assistir-Cuidar/Administrar individual é praticado por meio de procedimentos técnicos de comunicação e interação entre os sujeitos envolvidos com a assistência. O processo de trabalho em Saúde Coletiva, por sua natureza, pode apresentar diferentes objetos e finalidades e, neste estudo, manifestou-se no âmbito da Educação Política para a participação social dos sujeitos envolvidos com os serviços psiquiátricos públicos.

As entrevistas manifestam críticas ao processo de efetivação de reforma psiquiátrica que realiza mudanças, sobretudo, na estrutura física e administrativa, mas, mantém o trabalho no enfoque tradicional, na maioria dos serviços do CPMJ, revelando um processo de reforma lento, e contraditório, pois, no HD, o trabalho de enfermagem é revelador de um momento do processo de trabalho para a produção da inclusão social, contudo, dependente do trabalho da gestão que impõe limites ao trabalho inovador e interdita essa possibilidade aos demais serviços do Complexo.

Desse modo, o processo da reforma psiquiátrica em João Pessoa, vivencia um momento de transição, no qual o significado de transformação ainda não pode ser atribuído a todos os serviços, sendo o trabalho do HD o que mais avançou nesse sentido. A manutenção do trabalho conservador na maioria dos serviços do CPJM confere à refor-
O trabalho de enfermagem no hospital dia na perspectiva da reforma psiquiátrica em João Pessoa Paraíba 
Ana Teresa M.C. Silva Sônia Barros ma o caráter de atualização do modelo tradicio$n a l$, porque suas mudanças são superficiais, não atingindo a essência do modelo, o trabalho para a produção social da vida da pessoa com doença mental, conforme os depoimentos:

\begin{abstract}
O trabalho de enfermagem, apesar de suas especificidades, depende de outros trabalhos, principalmente do trabalho de administração e gerência do Complexo que centraliza tudo: é autoritário e tenta impedir a formação de espaços para se discutir a reforma. A equipe do HD questiona essas mudanças nas relações de trabalho, e outras mudanças que eles chamam de reforma, mas que não muda nada. (Aline)
\end{abstract}

O trabalho de enfermagem tem priorizado o acolhimento, a humanização das relações entre os colegas, entre os profissionais e os usuários; a discussão com a equipe de saúde do serviço; o atendimento à família, a orientação da importância da organização das famílias em entidades autônomas e fora dos muros do Complexo; a participação da família na assistência, nos eventos e a promoção de eventos para envolver a sociedade na nova proposta. (Mércia)

O trabalho da gente aqui é muito diferente do trabalho que eu fazia na Colônia. Lá, eu cumpria tarefa. Aqui eu compreendo porque preciso fazer as coisas. Às vezes eu faço coisas que nunca pensei fazer e, outras vezes, deixo de fazer o que pensei fazer porque, em certos momentos, eu sinto que posso resolver fazendo de outro jeito. Isso é muito gratificante, faz você pensar e gostar do que está fazendo. (Diva)

Nesses textos, os sujeitos manifestam uma mudança no modo de realizar o trabalho de enfermagem a partir da crítica ao modelo tradicional de assistência psiquiátrica, apresentada por Aline, na referência ao autoritarismo das relações sociais de trabalho a ser superado nas unidades de internação do CPMJ, para que se configure um novo modelo de assistência na perspectiva da reforma proposta.

A declaração de Diva sobre o novo trabalho no HD permite-lhe pensar e decidir sobre certas práticas, em oposição ao cumprimento de tarefas do modelo tradicional do trabalho de enfermagem - que permanece nos serviços tradicionais - reforça o reconhecimento da necessidade de consolidação do novo processo de trabalho desenvolvido no HD e de sua ampliação para as outras unidades de assistência à saúde mental, para uma aproximação com a possibilidade de uma reforma transformadora da assistência psiquiá- trica tradicional. Esse reconhecimento foi identificado, também, no depoimento de Mércia quando ela se refere às novas atividades em sua prática: o acolhimento, a humanização das relações entre os sujeitos implicados com a assistência em saúde mental e o envolvimento dos familiares com o serviço, além de afirmar a necessidade de organização e a participação social desse grupo.

No primeiro plano de análise sobre a concepção de reforma psiquiátrica, identificamos um tema comum em todos os discursos: mudar $o$ modo tradicional de assistir a pessoa com doença mental. Ao aprofundarmos a análise, procurando a qualidade atribuída a essa mudança, identificamos uma aproximação com a qualidade transformadora da assistência referida à reforma, no caráter das propostas de mudanças tanto no aspecto técnico-formal quanto no aspecto político do trabalho de enfermagem do HD, conforme os depoimentos:

A reforma psiquiátrica é uma tentativa de mudar, tanto a maneira de pensar, como a de executar a assistência aos doentes mentais. Durante séculos, esses pacientes foram excluídos, considerados perigosos, abandonados em hospitais que mais pareciam depósitos de lixo humano. Já era tempo de se pensar um novo jeito de resgatar, pelo menos, a condição de ser humano dos doentes mentais. Ainda estamos longe, mas, já foi dado o primeiro passo com o reconhecimento da necessidade de mudar essa realidade. (Mércia)

Não basta só pensar e falar em mudar, é preciso mudar, também, na prática. Veja a vida dos pacientes aqui, no Complexo: depois das mudanças, em nome da reforma, apesar de uma assistência mais humanizada, o espaço deles para circular foi reduzido nas Unidades fechadas. Isso não é um avanço, agora eles estão excluídos também no asilo. Que reforma é essa? Aqui, no Complexo, o serviço que consegue uma aproximação com a mudança de fato é o HD, porque, além de reconhecer que a reforma não é apenas a implantação de serviços substitutivos, trabalha com a mudança de atitude das pessoas que trabalham nesses serviços, diante do doente mental, da exclusão, do estigma e da qualidade de vida desses usuários. (Aline)

Além das concepções de reforma psiquiátrica, os depoimentos informam também, os elementos do processo de trabalho:

A Concepção de reforma psiquiátrica manifestou-se na compreensão da necessidade de mudança transformadora nas relações sociais de trabalho que implique o reconheci- 
mento dos direitos de cidadania do grupo acometido por doença mental; a possibilidade de reinserção social dessas pessoas e a efetiva substituição do hospital psiquiátrico tradicional por serviços substitutivos, como o HD e seu novo processo de trabalho.

A Concepção do objeto de trabalho apresentou-se no recorte, saúde/doença mental compreendido como um processo e um objeto socialmente construído, reconhecido nas relações sociais de trabalho no âmbito do atendimento das necessidades individuais e coletivas, conforme a referência de Aline à necessidade de novas relações de trabalho para a produção da vida biológica e social dos sujeitos que necessitam de assistência na área da saúde mental.

A Finalidade do trabalho de enfermagem revelou-se no processo de assistir/cuidar de pessoas com doença mental e de seus familiares. Ministrar cuidados de enfermagem no âmbito singular das necessidades individuais, e, no âmbito particular de atendimento à política nacional de saúde mental, buscando a relação com a totalidade da realidade social.

Entre os Instrumentos do trabalho de enfermagem, além de seu saber técnico especializado, para a assistência de enfoque da Saúde Individual, foram associados outros saberes pertinentes ao enfoque da Saúde Coletiva, como o saber da Educação e da Política Social, identificados na compreensão da importância de crítica da realidade e da participação política de todos os sujeitos implicados com a assistência à saúde mental, além de sua organização em movimentos sociais para ampliar o poder de conquistar suas reivindicações.

A análise do material empírico revelou qualidades de caráter e formal e político ${ }^{(a)}$ que o trabalho de enfermagem assume ${ }^{(12)}$. Essas qualidades orientam nossa análise para a construção de categorias empíricas de sentido transformador da assistência de enfermagem, no HD de João Pessoa na perspectiva da reforma psiquiátrica, porque os entrevistados operaram uma crítica ao modo dominante de assistência de enfermagem à pessoa com doença mental, nas instituições que compõem o Complexo Psiquiátrico, e associaram à qualidade formal de seu trabalho a importância de se considerar, também, o fomento à luta política de profissionais, usuários e seus familiares, por direitos e ampliação do espaço do poder.

Assim, os entrevistados articularam uma competência de qualidade técnico-formal com uma competência política, ao utilizar como instrumento de trabalho um saber que se revela na reflexão crítica sobre a realidade da assistência psiquiátrica que, no plano administrativo, propõe mudanças, apenas, nos novos serviços, como o HD, mantendo o asilo e o saber que o autoriza como instrumento do trabalho nos demais serviços, dificultando uma aproximação com a superação paradigmática porque, na maioria dos serviços, falta ao trabalho um potencial da qualidade transformadora, conforme o depoimento que afirma "o trabalho da gente aqui [no HD] é muito diferente do trabalho que eu fazia na Colônia". Vale salientar que a Colônia é um dos hospitais psiquiátricos tradicionais do CPJM, separado geograficamente dos demais serviços, apenas, por uma parede, contudo, mantendo uma distância de profundidade incalculável em relação à qualidade da assistência de enfermagem praticada no HD.

\section{CONSIDERAÇÕES FINAIS}

Este estudo permitiu-nos considerar que o referencial teórico-metodológico adotado possibilitou uma aproximação do objeto do estudo com os objetivos propostos, por ter-se mostrado adequado para a identificação da necessidade de se realizarem mudanças transformadoras no modelo tradicional de assistência psiquiátrica, nos serviços psiquiátricos públicos de João Pessoa, a partir dos processos de trabalho em saúde, entre os quais encontra-se o processo de trabalho de enfermagem.

A perspectiva da possibilidade de superação das relações sociais de trabalho autoritárias e segregadoras, que a proposta de uma reforma psiquiátrica suscita, implica um processo de trabalho de enfermagem cujos elementos apresentem potencial para articular a qualidade formal com a qualidade política, como um requisito que deve ancorar a construção de categorias empíricas potentes para explicar o significado que a reforma psiquiátrica assume e exprime nos processos de trabalho de enfermagem, da instituição investigada.

Os discursos manifestaram temas no reconhecimento da diferença entre transformar e atualizar o modelo tradicional, reafirmando a necessidade de superação do modelo de assistência psiquiátrica tradicional; a importância da participação social nesse processo e do reconhecimento da inter-relação entre as categorias, Trabalho de Enfermagem/Sociedade/Estado/Saúde Mental. Ou seja, há uma compreensão dos sujeitos entrevistados, de que o trabalho de enfermagem deve articular-se com outras práticas sociais para potencializar seu poder de transformação nos micro-espaços de sua atuação profissional.
O trabalho de enfermagem no hospital dia na perspectiva da reforma psiquiátrica em João Pessoa Paraíba (a) A qualidade, nos aspectos formal e político, foi estudada por Demo ${ }^{(12)}$. O autor afirma que a qualidade formal refere-se a instrumentos e métodos e diz respeito à seleção e montagem de instrumentos, sendo de muita utilidade para as abordagens de questões técnicas. Considera formal a qualidade que se apresenta como forma, capaz de ser replicada indefinidamente em casos previstos. A qualidade política refere-se a conteúdos e não se atém apenas aos meios ou aos instrumentos ou à finalidade, mas, a esse conjunto tomado historicamente; não se refere à forma, mas, à substância e somente os seres humanos a produzem, por isso o referido autor a conceitua, como: "a qualidade que trata dos conteúdos da vida humana e sua perfeição na arte de viver. Refere-se ao relacionamento do homem com a natureza, sobretudo através do trabaIho...onde inevitavelmente entra o horizonte ideológico e prático".

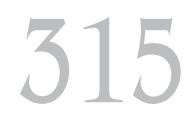

Rev Esc Enferm USP 2005; 39(3):310-6. 
Ana Teresa M.C. Silva Sônia Barros
Nesse sentido, as posições discursivas informaram a necessidade de crítica e negação das Instituições Totais, além da importância de se repensar as relações estabelecidas entre os sujeitos envolvidos com o trabalho em saúde mental para uma mudança transformadora da assistência psiquiátrica dominante. Assim, a assis- tência à saúde/saúde mental articula-se com o modo de produção dos serviços de saúde, organizado em sistemas financeiros, materiais e de recursos humanos, como a força de trabalho da enfermagem, e, em tecnologias, como os saberes e as práticas que se objetivam no seu processo de trabalho.

\section{REFERÊNCIAS}

(1) Marx K. O Capital. Rio de Janeiro: Brasiliense; 1996. Livro 1, v.1.

(2) Gonçalves RBM. Práticas de saúde: processos de trabalho e necessidades. São Paulo: Departamento de Medicina Preventiva da Faculdade de Medicina USP; 1992.

(3) Barros S. O louco, a loucura e a alienação institucional: o ensino de enfermagem sub-júdice. Taubaté: Cabral Editora Universitária; 2001.

(4) Amarante P, organizador. Loucos pela vida: a trajetória da reforma psiquiátrica no Brasil. Rio de Janeiro: Fiocruz; 1995.

(5) Relatório final da $1^{\text {a }}$ Conferência Nacional de Saúde Mental; 1987 jun 25-28; Rio de Janeiro. Brasília: Ministério da Saúde; 1988.

(6) Relatório final da $2^{\text {a }}$ Conferência Nacional de Saúde Mental; 1992 dez.1-4; Brasília. Brasília: Ministério da Saúde, 1994.
(7) Relatório final da $3^{\text {a }}$ Conferência Nacional de Saúde Mental; 2001 dez.11-15; Brasília. Brasília: Ministério da Saúde, 2002.

(8) Amarante P. O homem e a serpente: outras histórias para a loucura e a psiquiatria. Rio de Janeiro: Fiocruz; 1996.

(9) Chauí M. Convite à filosofia: $11^{\mathrm{a}}$ ed. São Paulo: Ática; 1995.

(10) Fiorin JL. Linguagem e ideologia. São Paulo: Ática; 1998.

(11) Castellanos BEP, Rodrigues AM, Almeida MCP, Rosa MTL, Mendes SASA. Os desafios da enfermagem para os anos 90 . In: Anais do $41^{\circ} \mathrm{Con}-$ gresso Brasileiro de Enfermagem; 1989. set. 2-7; Florianópolis. Florianópolis: UFSC; 1989. p. $147-69$.

(12) Demo P. Avaliação qualitativa. São Paulo: Cortez; 1998. 